\title{
Single-Mask SOI Fabrication Process for Linear and Angular Piezoresistive Accelerometers with On-Chip Reference Resistors
}

\author{
E. Jesper Eklund and Andrei M. Shkel \\ Department of Electrical Engineering and Computer Science \\ University of California, Irvine, CA 92697 \\ Email: eeklund@uci.edu, ashkel@uci.edu
}

\begin{abstract}
Piezoresistive accelerometers usually require separate photomasks for the fabrication of the proof mass, suspension, lightly doped piezoresistors, and highly doped conductors. This paper introduces a fabrication process that defines all components simultaneously using a single mask. By dry-etching SOI wafers that have a specific resistivity, piezoresistors can be defined and isolated from each other without the pn-junction normally required in piezoresistive sensors. The conductors are designed to be much wider than the piezoresistors and a free-standing proof mass is obtained by selectively removing the buried silicon oxide layer. Furthermore, reference resistors that provide the second half of a Wheatstone bridge are included on the silicon chip, eliminating the need for external resistors. Both single-axis and angular accelerometers have been fabricated and linear output characteristics are demonstrated when the single-axis devices are subjected to acceleration.
\end{abstract}

\section{INTRODUCTION}

Piezoresistive accelerometers are traditionally fabricated by doping selected areas of a wafer to achieve pn-junctions that are used to isolate the devices from the bulk substrate. Two separate doping steps are usually employed to obtain both highly doped conductors as well as lightly doped piezoresistors. Once the piezoresistors and conductors have been defined, additional fabrication steps are required to etch the suspension system as well as the free-standing proof mass [1]. At least four different photomasks are normally required when fabricating piezoresistive accelerometers.

A new method for fabricating piezoresistive accelerometers is proposed in this paper. This fabrication process is based on Silicon-on-Insulator (SOI) wafers. By choosing wafers with a particular resistivity, the need for doping steps can be eliminated from the fabrication process and the devices can be fabricated using a single photomask.

\section{PRINCIPLE OF OpERATION}

\section{A. Piezoresistivity}

Assume that a force is applied to the end of a long isotropic bar. The stress is then defined as the force divided by the crosssectional area of the bar. The resulting strain is the change in length divided by the initial length, $\Delta L / L_{0}$. As long as the strain is not too large, many solid materials behave like linear springs, meaning that the deformation is proportional to the applied force or acceleration.

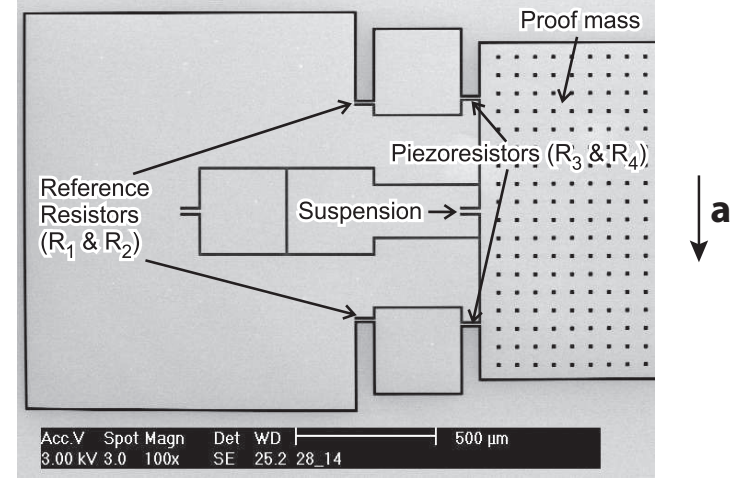

Fig. 1. Single-axis accelerometer. ${ }^{1}$

The piezoresistive effect causes a change in resistance, $\Delta R$, when a conductive material with initial electrical resistance $R_{0}$ is subjected to stress. Assuming that the stress in a piezoresistor is mainly in the longitudinal direction, the change in resistance $\Delta R$ due to a change in beam length $\Delta L$ is given by

$$
\frac{\Delta R}{R_{0}}=G F \frac{\Delta L}{L_{0}}
$$

where $R_{0}$ is the initial resistance, $L_{0}$ is the initial length, and $G F$ is the gage-factor. The gage-factor can be calculated as Young's modulus multiplied by the piezoresistive coefficient, which are both material constants.

\section{B. Accelerometer}

For a quasi-static accelerometer, the deflection at the center of the proof mass is

$$
x=\frac{a}{\left(2 \pi f_{n}\right)^{2}}
$$

where $a$ is the applied acceleration and $f_{n}$ is the natural frequency. For example, in the single-axis accelerometer in Fig. 1 , the proof mass deflects due to applied acceleration, $a$. The deflection leads to bending of the centrally located suspension

\footnotetext{
${ }^{1}$ Note that the proof-mass extends to the right beyond the displayed portion of the accelerometer.
} 
beam, in turn increasing the length of one of the piezoresistors by $|\Delta L|$ and decreasing the length of the other by $|\Delta L|$. This leads to increased resistance in the stretched piezoresistor and decreased resistance in the compressed piezoresistor, according to Equation (1). By detecting the change in resistance, the change in length of the piezoresistors can be calculated. The acceleration can then be obtained from Equation (2) by considering the geometry in Fig. 1 and relating $\Delta L$ to the deflection of the center of gravity of the proof mass, $x$.

The accelerometer in Fig. 1 is designed to emulate a Wheatstone bridge. Half of the bridge consists of piezoresistors that are attached to the proof mass $\left(R_{3}\right.$ and $\left.R_{4}\right)$ and the other two resistors are fixed reference resistors $\left(R_{1}\right.$ and $\left.R_{2}\right)$. Assuming the initial resistances are equal, the output voltage is $V_{\text {out }}=V_{\text {in }} \cdot \Delta R /\left(2 R_{0}\right)$.

\section{FABRICATION PROCESS}

The single-mask fabrication process depicted in Fig. 2 is based on SOI wafers with a specific resistivity. First, a layer of Shipley 1827 photoresist is spin-coated onto the wafer. The layout of the proof mass, suspension, thin piezoresistors, and wide conductors is then patterned before developing the photoresist. Next, deep-reactive ion etching (DRIE) is utilized to etch the device layer of the SOI wafers all the way to the buried silicon oxide. The wafer is then diced and the photoresist is stripped. Finally, selected parts of the silicon oxide layer is removed with Hydrofluoric (HF) acid and a free-standing proof mass is obtained. Areas without release holes define anchor points.

While the piezoresistors are attached to the proof mass, the reference resistors are anchored. Since the reference resistors are included on the chip and fabricated to be equal in size to the piezoresistors, all four resistors should have the same temperature properties. On the contrary, in many traditional piezoresistive sensors off-chip reference resistors are used, often requiring temperature compensation. Also, the proposed SOI fabrication process should allow for higher operational temperatures than sensors where pn-junctions are used to isolate the piezoresistors due to high leakage currents in pnjunctions above $150^{\circ} \mathrm{C}$ [2]. Note that while the proposed sensors will likely be operational up to about $300^{\circ} \mathrm{C}$, temperature compensation may still be required due to the temperature dependence of the piezoresistive coefficient.

Fig. 3 displays a close-up of one of the $2 \mu \mathrm{m}$ wide piezoresistors from the accelerometer in Fig. 1. The suspension beam is preferably much wider than the piezoresistors to assure that most of the bending occurs around the center of the device.

\section{FABRICATED ACCELEROMETERS}

\section{A. Linear Accelerometer}

The accelerometers are designed to emulate a Wheatstone bridge, as was illustrated in Fig. 1. In the linear accelerometer, a half-bridge configuration is used with both the piezoresistors ( $R_{3}$ and $R_{4}$ in Fig. 1) and reference resistors $\left(R_{1}\right.$ and $R_{2}$ in Fig. 1) included on the chip.

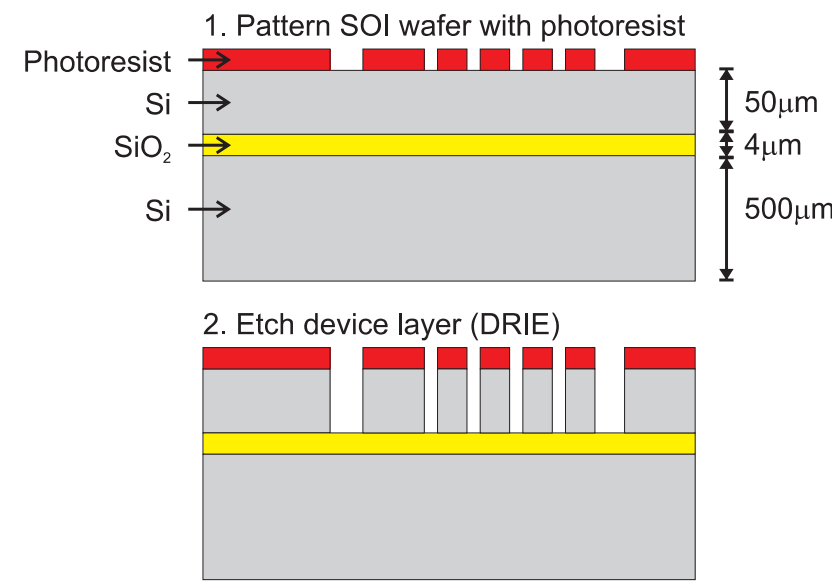

3. Remove photoresist and $\mathrm{SiO}_{2}$

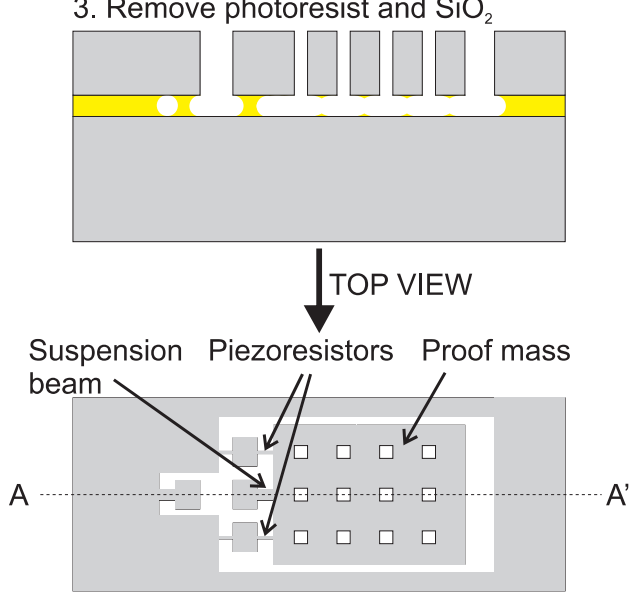

Fig. 2. SOI fabrication process.

Applied acceleration will cause bending of the centrally located suspension hinge, and will stretch one of the piezoresistors (increased resistance) and compress the other (decreased resistance). This in turn leads to an unbalanced bridge and $V_{\text {out }}$ is increased or decreased depending on the direction of the acceleration.

\section{B. Angular Accelerometer}

The angular accelerometer in Fig. 4 is configured as two separate full-bridges. All four resistors in the Wheatstone bridge are now piezoresistors. By properly connecting the bonding pads, the accelerometer can be configured to detect differential accelerations between two opposite sides. Thus, this device can be made immune to linear stimuli and should ideally only measure angular accelerations.

Table I demonstrates how an angular accelerometer responds to acceleration. For example, assume that acceleration is applied downward $(\Downarrow)$ in Fig. 4. The suspension beams parallel to the axis of acceleration are now much stiffer than the suspension beams perpendicular to the axis of acceleration. The longitudinal stiffness of a beam is $k_{l}=E h w / L$ and the transverse stiffness is $k_{t}=E h w^{3} /\left(4 L^{3}\right)$ [3], yielding a stiffness ratio of $k_{l} / k_{t}=4 L^{2} / w^{2}$, where $L$ is the length and 


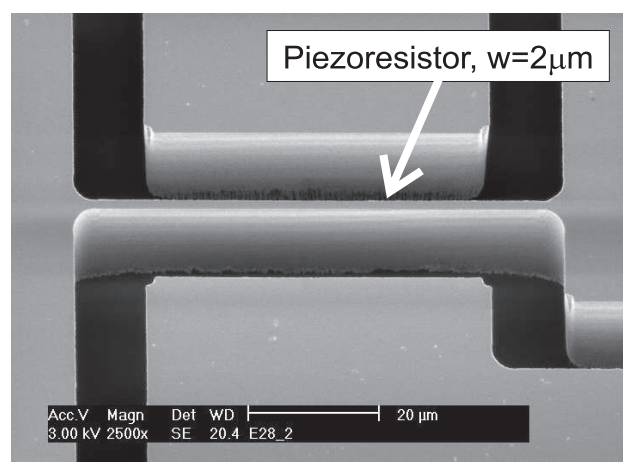

Fig. 3. Close-up of a piezoresistor.

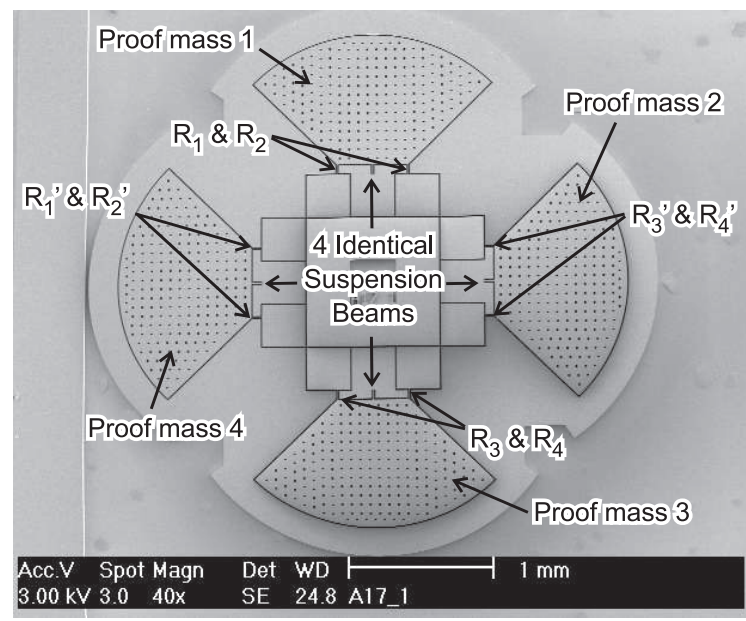

Fig. 4. Angular accelerometer.

$w$ is the width of the beam, and $L>>w$. Due to the large longitudinal stiffness, it can be assumed that the length of $R_{1}$, $R_{2}, R_{3}$ and $R_{4}$ will not significantly change (symbolized by a dash in Table I) when acceleration is applied downward. And since $R_{1}, R_{2}, R_{3}$ and $R_{4}$ do not change their resistance, the differential output from the bonding pads connected to the suspension beams, $V_{\text {out }}$, will not change.

TABLE I

Angular ACCElerometer Concept

\begin{tabular}{|c||c|c|c|c|c|c|c|c|c|c|}
\hline$a$ & $R_{1}$ & $R_{2}$ & $R_{3}$ & $R_{4}$ & $R_{1}^{\prime}$ & $R_{2}^{\prime}$ & $R_{3}^{\prime}$ & $R_{4}^{\prime}$ & $V_{\text {out }}$ & $V_{\text {out }}^{\prime}$ \\
\hline \hline$\Downarrow$ & - & - & - & - & $\downarrow$ & $\uparrow$ & $\downarrow$ & $\uparrow$ & - & - \\
\hline$\Uparrow$ & - & - & - & - & $\uparrow$ & $\downarrow$ & $\uparrow$ & $\downarrow$ & - & - \\
\hline$\Rightarrow$ & $\downarrow$ & $\uparrow$ & $\downarrow$ & $\uparrow$ & - & - & - & - & - & - \\
\hline$\Leftarrow$ & $\uparrow$ & $\downarrow$ & $\uparrow$ & $\downarrow$ & - & - & - & - & - & - \\
\hline$\circlearrowright$ & $\downarrow$ & $\uparrow$ & $\uparrow$ & $\downarrow$ & $\uparrow$ & $\downarrow$ & $\downarrow$ & $\uparrow$ & $\uparrow$ & $\downarrow$ \\
\hline$\circlearrowleft$ & $\uparrow$ & $\downarrow$ & $\downarrow$ & $\uparrow$ & $\downarrow$ & $\uparrow$ & $\uparrow$ & $\downarrow$ & $\downarrow$ & $\uparrow$ \\
\hline
\end{tabular}

Continuing the example when acceleration is applied downward $(\Downarrow), R_{1}^{\prime}$ and $R_{3}^{\prime}$ will be compressed and therefore decrease their resistance. At the same time, $R_{2}^{\prime}$ and $R_{4}^{\prime}$ will stretch and increase their resistance. But since the resistances will increase/decrease by equal amounts, the differential output voltage $V_{\text {out }}^{\prime}$ will not change and the Wheatstone bridge will stay balanced.

Similar arguments can be made for linear accelerations in other directions. However, when angular acceleration is applied, a change in output voltage is noted. For example, assume clockwise acceleration, which leads to compression (decreased resistance) of $R_{1}, R_{4}, R_{2}^{\prime}$ and $R_{3}^{\prime}$, and stretching (increased resistance) of $R_{2}, R_{3}, R_{1}^{\prime}$ and $R_{4}^{\prime}$. Since $R_{1}$ is decreased and $R_{2}$ is increased, the voltage at a point between these resistors increases. At the same time, the voltage at a point in between $R_{3}$ and $R_{4}$ decreases. $V_{\text {out }}$ will therefore increase. Simultaneously, $V_{\text {out }}^{\prime}$ will decrease due to the changes in $R_{1}^{\prime}$, $R_{2}^{\prime}, R_{3}^{\prime}$ and $R_{4}^{\prime}$. Similar arguments can be made for counterclockwise acceleration. Note that the increase/decrease of the output voltage is dependent on the input voltage. If desired, both outputs can be designed to have the same sign during angular acceleration by reversing the polarity of the input voltage of one of the two full-bridges in Fig. 4.

\section{EXPERIMENTAL RESUlTS}

A chip containing four different linear accelerometers is placed in a DIP-package and wire-bonded, as shown in Fig. 5. Aluminum wires are bonded straight onto the silicon, without using metal bonding pads, making this truly a single-mask fabrication process.

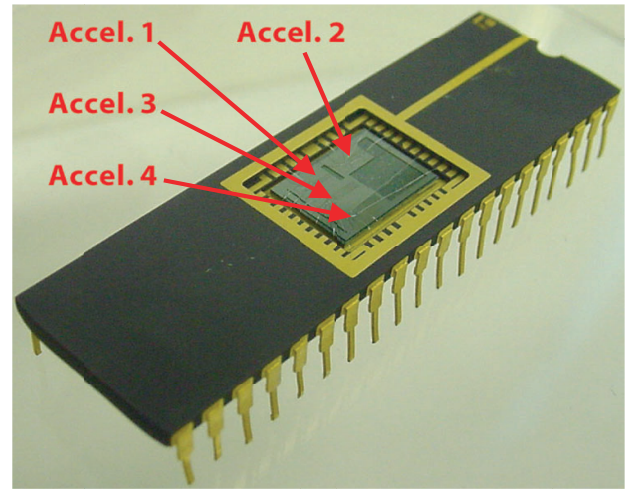

Fig. 5. Chip containing four different single-axis accelerometer in a DIPpackage.

The packaged accelerometers are positioned $26 \mathrm{~cm}$ from the center of a rate table, which has a maximum angular velocity of $1300^{\circ} / \mathrm{s}$. The velocity is increased in $100^{\circ} / \mathrm{s}$ increments from $400^{\circ} / \mathrm{s}$ to $1300^{\circ} / \mathrm{s}$, yielding accelerations from approximately $1 G$ to $13 G$. The same test is repeated with the accelerometer turned $180^{\circ}$ and positioned $24 \mathrm{~cm}$ from the center of the rate table, yielding accelerations from approximately $-1 G$ to $-13 G$. The experimental setup is shown in Fig. 6.

Fig. 7 shows a linear output from $-13 G$ to $+13 G$ when $V_{i n}=5 \mathrm{~V}$ is applied. The sensitivity is $0.8 \mathrm{mV} / \mathrm{G}$. The offset of $19 \mathrm{mV}$ is due to fabrication imperfections that lead to a slightly unbalanced Wheatstone bridge. This offset can be reduced with off-chip resistors, although increased temperature 


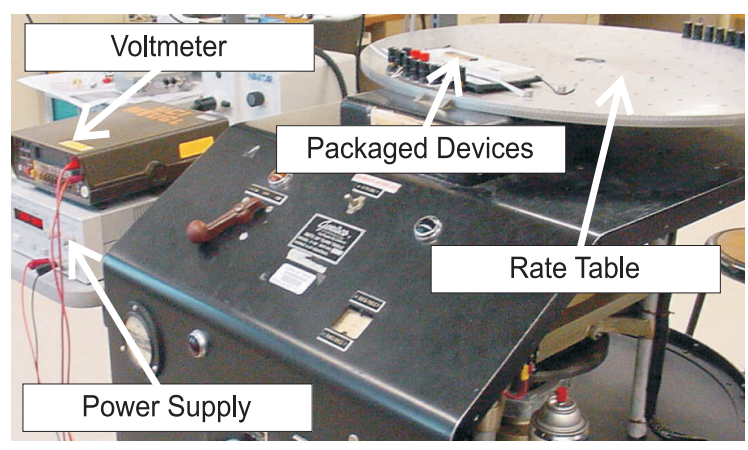

Fig. 6. Experimental setup.

dependence is then expected. Instead, signal-processing of the output is preferably used to eliminate the voltage offset.

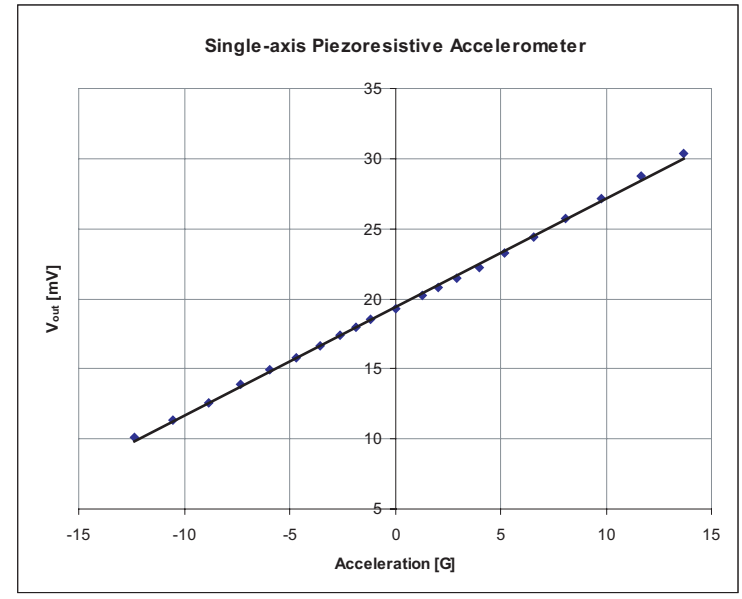

Fig. 7. Acceleration measurements on rate-table.

Next, a Dytran 3030A reference accelerometer is mounted in close proximity to the single-axis accelerometer to measure the time domain response. The mounting platform is then placed on a shaker. Fig. 8 displays the output from both the reference accelerometer and the single-mask piezoresistive accelerometer at $1 \mathrm{kHz}$. Both outputs were $\mathrm{AC}$ coupled to eliminate the offset voltage. The sensitivity of the reference accelerometer is $10 \mathrm{mV} / \mathrm{G}$ and the maximum output is about $520 \mathrm{mV}$, yielding a maximum acceleration of $52 G$. The single-mask piezoresistive accelerometer outputs approximately $40 \mathrm{mV}$ for this acceleration, which is consistent with the sensitivity of $0.8 \mathrm{mV} / \mathrm{G}$, previously obtained from Fig. 7 .

The natural frequency of the single-axis accelerometer is estimated to be approximately $10 \mathrm{kHz}$. However, this has not yet been experimentally verified due to the limited frequency range of the shaker. Furthermore, while the angular accelerometer was conceptually described in a previous section, it needs to be thoroughly characterized before anything can be stated about its performance.

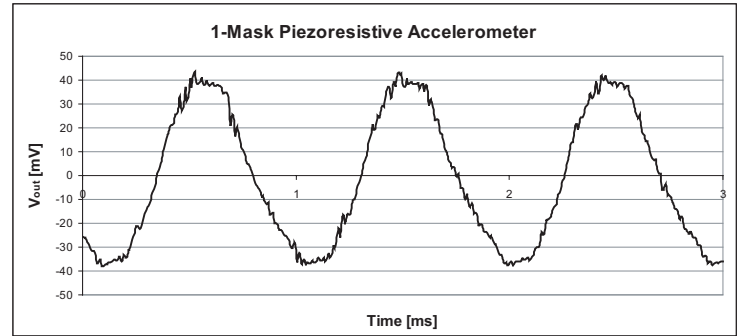

(a)

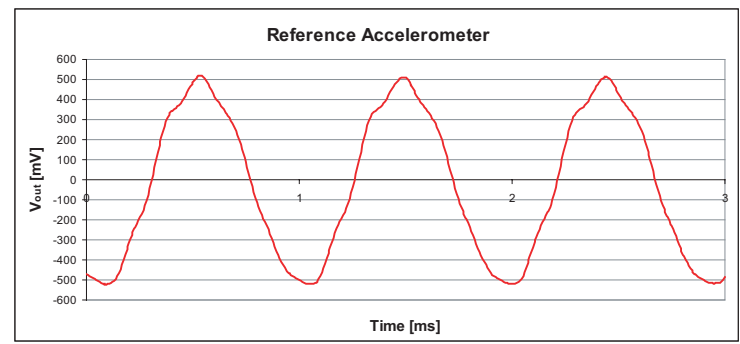

(b)

Fig. 8. Time domain response at $1 \mathrm{kHz}$ and $52 \mathrm{G}$ excitation for (a) single-mask piezoresistive accelerometer and (b) reference accelerometer.

\section{CONCLUSION}

A single-mask fabrication process for piezoresistive accelerometers has been introduced. This process significantly reduces the number of required fabrication steps when compared to conventional piezoresistive sensors. Both single-axis and angular accelerometers have been fabricated with this fabrication process, and linear output characteristics were demonstrated when a single-axis accelerometer was subjected to acceleration.

\section{ACKNOWLEDGMENT}

This research was supported by VIP Sensors Grant 44253119919 and UC Discovery Grant 482530-59829.

\section{REFERENCES}

[1] L. Roylance and J. Angell, "A batch-fabricated silicon accelerometer," IEEE Trans. Electron Devices, vol. ED-26, no. 12, pp. 1911-1917, 1979.

[2] M. Werner and W. Fahrner, "Review on materials, microsensors, systems, and devices for high-temperature and harsh-environment applications," IEEE Trans. Ind. Electron., vol. 48, no. 2, pp. 249-257, 2001.

[3] W. Young and R. Budynas, Roark's Formulas for Stress and Strain, 7th ed. McGraw-Hill Professional, 2001. 